\section{Pinpointing triggers in MS}

\section{By Lev Osherovich, Senior Writer}

Two recent papers identify key players in the early stages of multiple sclerosis, opening up a large class of potential therapeutic targets.

A new mouse model described in Nature Medicine has helped researchers uncover the identity of the immune cells responsible for brain inflammation, pointing to Th17 helper T cells as a leading suspect in triggering MS. ${ }^{1}$ The findings bolster ZymoGenetics Inc.'s rationale for pursuing its soluble interleukin-17 receptor in MS.

Separately, a survey of proteins found in diseased brain tissue points to an unexpected role for blood clotting proteins in triggering immunological attack on nerve tissue. ${ }^{2}$ The results, together with those from the new mouse model, point to proteins in the coagulation cascade as key effectors of Th17-mediated inflammation. The targets, which were published in Nature, could open MS as an indication for companies developing certain anticoagulants.

In the Nature Medicine paper, University of Washington researchers developed an improved version of the standard mouse model of MS called experimental autoimmune encephalomyelitis (EAE) to tease out the cause of brain inflammation in the disease. The conventional EAE model has many features in common with MS, but unlike the human disease it is largely confined to the spinal cord, making brain-specific features of MS difficult to address.

Beginning with a mutant mouse strain prone to MS-like brain inflammation, the researchers identified a brain-derived protein epitope found only in abnormally sick animals. The research team, led by Joan Goverman, professor of immunology at the university, then used the epitope to identify Th17 helper T cells as the immune cells that trigger mouse MS. This finding jibes with a spate of recent reports implicating Th17 cells in MS.,4

Th1 helper T cells and Th17 cells were already known to kick-start spinal nerve tissue inflammation in the EAE model. However, the specific identity of the cells that travel beyond the spinal cord and into the brain was unknown. Goverman's team found that Th17 cells preferentially responded to the epitope and were overrepresented in inflamed mouse brains relative to Thl cells.

"One of the key findings in our paper was that you could alter the ratios of Th1 and Th17 cells to get brain inflammation," Goverman told SciBX. Th1 cells appear to secrete cytokines that dampen Th17 activation, she said, but the problem is Th1 cells do not enter the brain as efficiently as do Th17 cells.

To prove that Th17 cells are indeed responsible for inflammation, Goverman's team injected healthy mice with preactivated Th17 cells and observed rapid inflammation of both brain and spinal cord. Moreover, blocking interleukin-17 (IL-17)-the inflammatory cytokine secreted by activated Th17 cells-prevented inflammation in the brain but not in the spinal cord.

Thus, the mouse model data suggest Th17 cells could be the missing link in the start of infiltration of the brain by the immune system (see Figure 1, "Th17 cells and coagulation factors in multiple sclerosis"). According to this theory, Th17 cells may be coordinating the erroneous attack on neurons by neutrophils, macrophages and other inflammatory immune cells.

Nicolle Onetto, SVP and chief medical officer at ZymoGenetics, told SciBX that the Goverman paper is an "extremely encouraging result for IL-17." ZymoGenetics and partner Merck Serono S.A. have developed IL-17RC, a soluble form of the IL-17 receptor that binds up blood-borne IL-17, reducing inflammation in preclinical models.

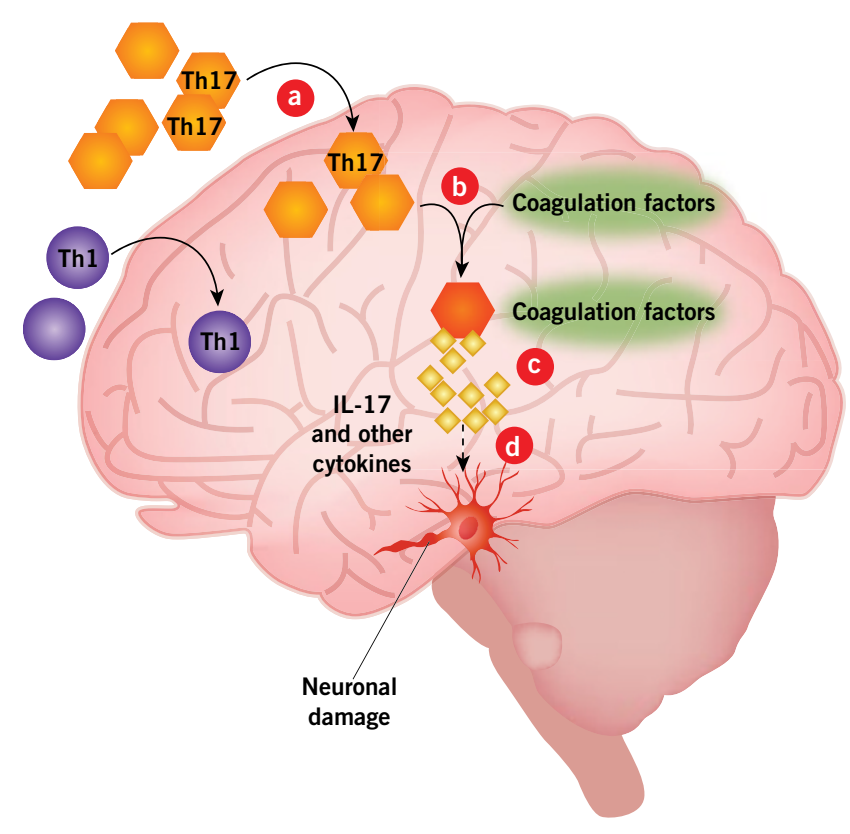

Figure 1. Th17 cells and coagulation factors in multiple sclerosis. New findings by Stromnes et al. and Han et al. suggest a possible sequence for early events in multiple sclerosis in the brain, where Th17 cells may play a more dominant role than Th1 cells in inflammation. Th17 cells, more so than Th1 cells, are drawn to the brain [a], interact with blood vessel-associated coagulation factors and become activated [b]. Once activated, these cells release cytokines such as interleukin-17 (IL-17) that recruit other immune cells and promote inflammation [c], leading to the destruction of the myelin coat of neurons [d]. Th17 cell-specific proteins, coagulation factors and cytokines such as IL-17 are thus potential therapeutic targets for MS. Here, coagulation factors appear to play a different role than their known functions in blood clotting. 
ZymoGenetics is contemplating an IND filing for IL-17RC to treat MS.

Frank Bennett, SVP of research at Isis Pharmaceuticals Inc., noted that Goverman's findings on Th17 cells could lead to a useful biomarker for companies seeking to understand how their compounds work. Isis is developing ATL1102, an antisense inhibitor of integrin $\alpha_{4} \beta_{1}$ (VLA-4), under a deal with Teva Pharmaceutical Industries Ltd. The compound is in Phase II testing in MS.

"In thinking about how to modulate MS from an immunological perspective, this finding gives you more fine tuning, allowing you to be more directed," said Bennett. For example, he said, the improved mouse EAE model could allow Isis to determine whether ATL1102 selectively targets VLA-4 on Th17 cells rather than helper T cells in general.

Lawrence Steinman, professor of immunology at Stanford University, said the Nature Medicine paper is "a significant improvement in the EAE model" that will be useful in studying the MS disease process. "This paper is very beautiful because it shows that different regions of the nervous system have different requirements for entry," he added.

Goverman told SciBX that she had not filed patents on her discoveries.

Steinman was senior author on the Nature paper, in which his team used mass spectrometry to identify the proteins in MS-stricken brains. He said the results led to a "goldmine" of therapeutic candidates.

The researchers compared the protein profile of healthy brains with damaged areas in the brains of MS patients and found hundreds of proteins unique to various kinds of MS lesions. Over half of the proteins were previously uncharacterized and thus are potentially new targets.

"I've been in the game for quite a few years and it's astonishing that there are so many proteins without [known] functions that turn out to be important here," Steinman told SciBX. He added that a second paper describing a new class of MS-associated proteins is in preparation.

The Nature paper focused on the low-hanging fruit-proteins with known functions that are targeted by approved drugs. This category included five coagulation factors, including thrombin and protein $\mathrm{C}$ inhibitor, which were overabundant in chronic active MS plaques-the kind most closely associated with inflammation.

Mice treated with hirudin, a generic antithrombotic, or Eli Lilly and Co.'s Xigris drotrecogin alfa activated protein C, were resistant to EAE compared with untreated controls.

"This work clearly implicates the coagulation cascade as a therapeutic target," said Goverman, referring to Steinman's study. She cautioned that targeting these proteins with MS therapeutics could prove challenging, but not impossible. "You can't just eliminate coagulation in people, but that doesn't mean that there won't be a way to [selectively] do this."

The good news is that the clot-busting activity of Xigris was not required for efficacy in MS. Steinman's group found that a mutant form of Xigris that does not prevent clotting could still protect mice against EAE. This suggests that in the MS setting, coagulation factors modulate helper $\mathrm{T}$ cell activation independently of their role in blood clotting, Steinman told SciBX.

"It's very impressive that coagulation could have such a dramatic effect on the animal model," said Isis' Bennett. "This isn't a therapeutic approach that you would have thought of independently of the data."

Mark Krantz, VP of scientific affairs at BioMS Medical Corp., thinks both studies are significant developments, but cautioned "it's easier to treat EAE than real MS. I don't get really excited by animal studies, but if there are existing drugs that already have approval, it's pretty easy to get into a Phase I trial."

BioMS is running Phase III trials of MBP8298, a synthetic myelin basic protein peptide designed to elicit a protective immune response to block the onset of MS in certain genetically susceptible patients. Last year, the company signed a worldwide licensing agreement with Eli Lilly for future R\&D, manufacturing and marketing of MBP8298.

Goverman said an important next step will be to figure out how coagulation factors stimulate helper $\mathrm{T}$ cell activation or enable their passage across the blood-brain barrier, a key early event in MS. Steinman said this may occur through physical contact between helper $\mathrm{T}$ cells and blood vessels at the edge of the brain, leading to adhesion and activation.

Cell-adhesion molecules, such as VLA-4, are known players in MS. In addition to Isis' compound, Tysabri natalizumab, a monoclonal antibody marketed for MS by Elan Corp. plc and Biogen Idec Inc., also targets VLA-4.

Steinman, whose lab discovered the role of VLA-4 in MS, speculated that anticoagulants could block helper T cell adhesion as effectively as Tysabri, but without the potential for immunological sequelae. ${ }^{5,6}$ In addition, the helper $\mathrm{T}$ cell surface proteins that bind to coagulation factors could make good therapeutic targets.

Steinman said Stanford has filed patent applications covering the discoveries in the Nature paper, in addition to other MS-related proteins identified in the mass spectrometry study. Steinman is a cofounder of Bayhill Therapeutics Inc. and a member of the company's board of directors and chairman of its scientific advisory board.

Bayhill is tackling MS and other autoimmune diseases with a DNA vaccine that promotes the immune system's tolerance to self-antigens, a technology that was initially developed in Steinman's lab and licensed by Stanford to the company under a 2002 agreement. However, Steinman told SciBX that Bayhill's rights do not include the "work on proteomics or the intersection of coagulation with inflammation."

\section{REFERENCES}

1. Stromnes, I.M. et al. Nat. Med.; published online Feb. 17, 2008; doi:10.1038/nm1715

Contact: Joan M. Goverman, Department of Immunology, University of Washington, Seattle, Wash.

e-mail: goverman@u.washington.edu

2. Han, M.H. et al. Nature; published online Feb. 17, 2008; doi:10.1038/ nature06559

Contact: Lawrence Steinman, Department of Pathology (Neuropathology), Stanford University, Stanford, Calif. e-mail: steinman@stanford.edu

3. Kebir, H. et al. Nat. Med. 13, 1173-1175 (2007)

4. Steinman, L. Nat. Med. 13, 139-145 (2007)

5. Maggos, C. BioCentury 13(11), A1; March 7, 2005

6. Usdin, S. BioCentury 14(12), A1; March 13, 2006

COMPANIES AND INSTITUTIONS MENTIONED

Bayhill Therapeutics Inc., Palo Alto, Calif.

Biogen Idec Inc. (NASDAQ:BIIB), Cambridge, Mass.

BioMS Medical Corp. (TSX:MS), Edmonton, Alberta, Canada

Elan Corp. plc (NYSE:ELN), Dublin, Ireland

Eli Lilly and Co. (NYSE:LLY), Indianapolis, Ind.

Isis Pharmaceuticals Inc. (NASDAQ:ISIS), Carlsbad, Calif.

Merck Serono S.A., Geneva, Switzerland

Stanford University, Stanford, Calif.

Teva Pharmaceutical Industries Ltd. (NASDAQ:TEVA), Jerusalem, Israel

University of Washington, Seattle, Wash.

ZymoGenetics Inc. (NASDAQ:ZGEN), Seattle, Wash. 